\title{
Advances in Computing the Nonabelian Tensor Square of Polycyclic Groups
}

\author{
ROBERT FITZGERALD MORSE
}

The nonabelian tensor square $G \otimes G$ of the group $G$ is the group generated by the symbols $g \otimes h$, where $g, h \in G$, subject to the relations

$$
g g^{\prime} \otimes h=\left({ }^{g} g^{\prime} \otimes{ }^{g} h\right)(g \otimes h) \quad \text { and } \quad g \otimes h h^{\prime}=(g \otimes h)\left({ }^{h} g \otimes{ }^{h} h^{\prime}\right)
$$

for all $g, g, h, h^{\prime} \in G$, where ${ }^{g} g^{\prime}=g g^{\prime} g^{-1}$ is conjugation on the left. Following the work of C. Miller [18], R. K. Dennis in [10] introduced the nonabelian tensor square which is a specialization of the more general nonabelian tensor product independently introduced by R. Brown and J.-L. Loday [6].

By computing the nonabelian tensor square we mean finding a standard or simplified presentation for it. In the case of finite groups, the definition of the nonabelian tensor square gives a finite presentation that can be simplified using Tietze transformations. This simplified presentation can then be examined to determine the nonabelian tensor square. This was the approach taken in [3], in which the nonabelian tensor square was computed for each nonabelian group of order up to 30. Creating a presentation from the definition of the nonabelian tensor square, simplifying it using Tietze transformations and computing a structure description from the simplified presentation can be implemented in few lines of GAP [16]. However, this strategy does not scale well to finite groups $G$ having order greater than 100 since the initial presentation has $|G|^{2}$ generators and $2|G|^{3}$ relations.

The most general method for computing the nonabelian tensor square uses the notion of a crossed pairing (see [3]). Let $G$ and $L$ be

2000 Mathematics Subject Classification. 20F18, 20J06.

This paper is based on my talk at Groups in Galway, National University of Ireland, Galway, 13 May 2005. 
groups. We call the mapping $\Phi: G \times G \rightarrow L$ a crossed pairing if for all $g, g^{\prime}, g^{\prime \prime} \in G$ we have

$$
\Phi\left(g g^{\prime}, g^{\prime \prime}\right)=\Phi\left({ }^{g} g^{\prime},{ }^{g} g^{\prime \prime}\right) \Phi\left(g, g^{\prime \prime}\right)
$$

and

$$
\Phi\left(g, g^{\prime} g^{\prime \prime}\right)=\Phi\left(g, g^{\prime}\right) \Phi\left(g^{\prime} g, g^{g^{\prime}} g^{\prime \prime}\right) .
$$

The crossed pairing $\Phi$ lifts to a homomorphism $\Phi^{*}: G \otimes G \rightarrow L$ such that $\Phi^{*}\left(g \otimes g^{\prime}\right)=\Phi\left(g, g^{\prime}\right)$ for all $g, g^{\prime} \in G$.

To compute the nonabelian tensor square of a group $G$, we conjecture a group $L$ that we believe is isomorphic to $G \otimes G$ and construct a mapping $\Phi: G \times G \rightarrow L$. We then show that $\Phi$ is a crossed pairing and that the homomorphism $\Phi^{*}$ is actually an isomorphism. This method has been used to compute the nonabelian tensor square of nilpotent of class 2 groups [1], [21], infinite metacyclic groups [4], and the free 2-Engel groups [5], [9]. When the nonabelian tensor square is not abelian, constructing the function $\Phi$ and showing it is a crossed pairing can be very difficult. For example, computing the nonabelian tensor square of the free 2-Engel groups required computer assistance to symbolically manipulate the exponent functions that arise in proving the mapping $\Phi$ is a crossed pairing (see [5] and [9]). The crossed pairing method is an ad hoc method at best and does not lead to an algorithm for computing the nonabelian tensor square. To use this method one essentially has to know the answer a priori. However, it has been the only method available until now for computing the nonabelian tensor square of infinite groups.

The following group construction is found in [20]. Let $G$ and $G^{\varphi}$ be isomorphic groups via $\varphi: g \mapsto g^{\varphi}$ for all $g \in G$. Define the group $\nu(G)$ to be

$$
\begin{aligned}
\nu(G)=\left\langle G, G^{\varphi}\right|{ }^{k}\left[g, h^{\varphi}\right] & =\left[{ }^{k} g,\left({ }^{k} h\right)^{\varphi}\right] \\
& \left.={ }^{k^{\varphi}}\left[g, h^{\varphi}\right] \forall g, h, k \in G\right\rangle,
\end{aligned}
$$

where the commutator $[x, y]$ is defined to be $x y x^{-1} y^{-1}$. The significance of this group construction to the nonabelian tensor square is found in the following proposition.

Proposition 1 ([20]). Let $G$ be a group. The map $\rho: G \otimes G \rightarrow$ $\left[G, G^{\varphi}\right] \triangleleft \nu(G)$ defined by $\rho(g \otimes h)=\left[g, h^{\varphi}\right]$ is an isomorphism.

The following structural results were also obtained. 
Theorem 2 ([20]). Let $G$ be a group.

(i) If $G$ is finite then $\nu(G)$ is finite.

(ii) If $G$ is a finite p-group then $\nu(G)$ is a finite p-group.

(iii) If $G$ is nilpotent of class $c$ then $\nu(G)$ is nilpotent of class at most $c+1$.

(iv) If $G$ is solvable of derived length $d$ then $\nu(G)$ is solvable of derived length at most $d+1$.

We can use Proposition 1 and Theorem 2 to compute the nonabelian tensor square of a finite group $G$. We create the finite presentation for $\nu(G)$ given by (1). By Theorem 2 we know that $\nu(G)$ is finite and hence we can find a concrete representation of $\nu(G)$ and compute the subgroup $\left[G, G^{\varphi}\right]$, which by Proposition 1 is isomorphic to $G \otimes G$. In general, a concrete representation of $\nu(G)$ can be found by coset enumeration and in the special cases when $G$ is a $p$-group or is nilpotent one can use a $p$-quotient or nilpotent quotient algorithm to find a concrete representation of $\nu(G)$. The presentation given by (1) is an improvement on the presentation given by the definition of the nonabelian tensor square. If $G$ has the presentation $\langle X \mid R\rangle$ then $\nu(G)$ has $2|X|$ generators and $2\left(|R|+|G|^{3}\right)$ relations.

The group $\nu(G)$ can be found in the language of crossed modules [15] and its application for computing the nonabelian tensor product can be found in [12]. The next theorem specializes the main result in [12] to the nonabelian tensor square.

Theorem 3 ([12]). Let $G$ be a group. Then

$$
\nu(G) \cong G * G^{\varphi} /\langle J\rangle \cong((G \otimes G) \rtimes G) \rtimes G,
$$

where $J$ is a normal generating set and

$$
J=\left\{{ }^{x}\left[g, h^{\varphi}\right]\left[{ }^{x} h^{\varphi},{ }^{x} g\right]\right\}
$$

for $x \in G * G^{\varphi}$ and $g, h \in G$.

The presentation of $\nu(G)$ in Theorem 3 is infinite. The following theorem gives us a finite presentation of $\nu(G)$ when $G$ satisfies certain finiteness conditions.

Theorem 4 ([12]). Let $G$ be a group generated by the set $X$. Set $D$ to be the union of a generating set of $Z(G)$, the center of $G$, and a transversal of $Z(G)$ in $G$. Then $\nu(G) \cong G * G^{\varphi} /\left\langle J^{\prime}\right\rangle$, where

$$
J^{\prime}=\left\{{ }^{z}\left[g, h^{\varphi}\right]\left[{ }^{z} h^{\varphi},{ }^{z} g\right]\right\} \quad(\text { for } g, h \in X \text { and } z \in D)
$$

is a normal generating set. 
It follows from Theorem 4 that if $G=\langle X \mid R\rangle$ then $\nu(G)$ has a presentation with $2|X|$ generators and $2|R|+|X|^{2} \cdot|D|$ relations. If $G$ is finite then this presentation is significantly smaller than the presentation for $\nu(G)$ given in (1).

Russell Blyth, Joanne Redden and I [9] had just completed computing the nonabelian tensor square of the free 2-Engel groups using the crossed pairing method and wanted to extend our results to the free nilpotent groups of class 3 . The complexity of the crossed pairing method in the 2-Engel case suggested we should look for a different approach to the new problem. It follows from Theorem 4 and Theorem 2 that if $G$ is a finitely generated nilpotent group of class $c$ then $\nu(G)$ is a finitely generated nilpotent group of class at most $c+1$. Hence we started investigating the group $\nu(G)$ as a possible way to compute $G \otimes G$ for $G$ a free nilpotent group of class 3 and rank $n$. The following specialization of a result found in [17] pushed us to consider the more general class of polycyclic groups.

Theorem 5 ([17]). Let $G$ be a polycyclic group. Then $\nu(G)=G *$ $G^{\varphi} /\left\langle J^{\prime \prime}\right\rangle$, where the subgroup $\left\langle J^{\prime \prime}\right\rangle$ is normally generated by the words $\mathfrak{g}\left[g_{i}, g_{j}^{\varphi}\right] \mathfrak{g}^{-1}\left[{ }^{\mathfrak{g}} g_{i},\left({ }^{\mathfrak{g}} g_{j}\right)^{\varphi}\right]^{-1} \quad$ and $\quad \mathfrak{g}^{\varphi}\left[g_{i}, g_{j}^{\varphi}\right]\left(\mathfrak{g}^{\varphi}\right)^{-1}\left[{ }^{\mathfrak{g}} g_{i},\left({ }^{\mathfrak{g}} g_{j}\right)^{\varphi}\right]^{-1}$, where $g_{i}, g_{j}$ are elements of a generating set $\mathcal{G}$ of $G$ and $\mathfrak{g}$ is an element of a polycyclic generating sequence $\mathfrak{G}$ for $G$.

The following results are part of ongoing joint work [8].

Theorem 6. Let $G$ be a polycyclic group. Then $G \otimes G$ and $\nu(G)$ are polycyclic.

Corollary 7. Let $G$ be a polycyclic group with a polycyclic generating sequence $\mathfrak{g}_{1}, \ldots, \mathfrak{g}_{k}$. Then the subgroup $\left[G, G^{\varphi}\right]$ of $\nu(G)$ is generated by

for $1 \leq i, j, \leq k, i \neq j$, where

$$
\left\{\left[\mathfrak{g}_{i}, \mathfrak{g}_{i}^{\varphi}\right],\left[\mathfrak{g}_{i}^{\epsilon},\left(\mathfrak{g}_{j}^{\varphi}\right)^{\delta}\right]\right\}
$$

$$
\epsilon=\left\{\begin{array}{ll}
1, & \text { if }\left|\mathfrak{g}_{i}\right|<\infty \\
\pm 1 & \text { if }\left|\mathfrak{g}_{i}\right|=\infty
\end{array} \quad \delta= \begin{cases}1, & \text { if }\left|\mathfrak{g}_{j}^{\varphi}\right|<\infty \\
\pm 1 & \text { if }\left|\mathfrak{g}_{j}^{\varphi}\right|=\infty\end{cases}\right.
$$

Theorems 5 and 6 and Corollary 7 provide the theoretical support for an algorithm to compute the nonabelian tensor square of any polycyclic group. Let $G$ be a polycyclic group with generating set $\mathcal{G}$ and polycyclic generating sequence $\mathfrak{G}$. Then by Theorem 5 we can construct a finite presentation of $\nu(G)$. It follows from Theorem 6 
that $\nu(G)$ is polycyclic. Using a polycyclic quotient algorithm, we can determine a polycyclic presentation of $\nu(G)$, which gives us the ability to compute subgroups in $\nu(G)$. In particular we can compute the subgroup $\left[G, G^{\varphi}\right] \cong G \otimes G$ using the generating set given in Corollary 7.

This algorithm has been implemented in GAP using the Polycyclic package [14] to compute with infinite and finite polycyclic groups and the IPCQ package [11] to compute the polycyclic quotient of a finitely presented group. The IPCQ package is experimental and is not yet able to find the polycyclic presentation for $\nu(G)$ for $G$ any nonnilpotent infinite polycyclic group we have tried to date. However, if $G$ is nilpotent then we can effectively find a nilpotent presentation for $\nu(G)$ using the nq package [19]. The nonabelian tensor square of the free 2-Engel group of rank 3 was computed using crossed pairings in [5]. Using our GAP implementation the nonabelian tensor square of this group can be found in a few seconds.

It must be noted that the polycyclic presentation of the nonabelian tensor square given by the algorithm above for a nilpotent group $G$ must be examined to find structural information about $G \otimes G$. Routines exist in GAP to determine the nilpotency class and find the torsion subgroup of polycyclic groups. However, other structural information, such as whether the group is a direct product, can only be found by closely examining the polycyclic presentation given for $G \otimes G$.

Finding a polycyclic presentation for $\nu(G)$ is not necessary to find information about $\left[G, G^{\varphi}\right]$. A commutator calculus, which is equivalent to working in the nonabelian tensor square, was developed in [20]. The following lemmas summarize part of this calculus.

Lemma 8 ([20]). Let $G$ be a group. The following relations hold in $\nu(G)$ :

(i) ${ }^{\left[g_{3}, g_{4}^{\varphi}\right]}\left[g_{1}, g_{2}^{\varphi}\right]={ }^{\left[g_{3}, g_{4}\right]}\left[g_{1}, g_{2}^{\varphi}\right]$ and $\left[g_{3}^{\varphi}, g_{4}\right]\left[g_{1}, g_{2}^{\varphi}\right]={ }^{\left[g_{3}, g_{4}\right]}\left[g_{1}, g_{2}^{\varphi}\right]$ for all $g_{1}, g_{2}, g_{3}, g_{4} \in G$;

(ii) $\left[g_{1}^{\varphi}, g_{2}, g_{3}\right]=\left[g_{1}, g_{2}, g_{3}^{\varphi}\right]=\left[g_{1}^{\varphi}, g_{2}, g_{3}^{\varphi}\right]$ and $\left[g_{1}, g_{2}^{\varphi}, g_{3}\right]=\left[g_{1}^{\varphi}, g_{2}^{\varphi}, g_{3}\right]=\left[g_{1}, g_{2}^{\varphi}, g_{3}^{\varphi}\right]$ for all $g_{1}, g_{2}, g_{3} \in G ;$

(iii) $\left[g, g^{\varphi}\right]$ is central in $\nu(G)$ for all $g \in G$;

(iv) $\left[g_{1}, g_{2}^{\varphi}\right]\left[g_{2}, g_{1}^{\varphi}\right]$ is central in $\nu(G)$ for all $g_{1}, g_{2} \in G$;

(v) $\left[g, g^{\varphi}\right]=1$ for all $g \in G^{\prime}$. 
Lemma 9 ([20]). Let $a, b$ and $x$ be elements of a group $G$ such that $[x, a]=1=[x, b]$. Then in $\nu(G)$,

$$
\left[a, b, x^{\varphi}\right]=1=\left[[a, b]^{\varphi}, x\right] .
$$

Lemma 10 ([20]). Let $x$ and $y$ be elements of a group $G$ such that $[x, y]=1$. Then in $\nu(G)$,

(i) $\left[x^{n}, y^{\varphi}\right]=\left[x, y^{\varphi}\right]^{n}=\left[x,\left(y^{\varphi}\right)^{n}\right]$ for all integers $n$;

(ii) If $x$ and $y$ are torsion elements of orders o(x) and $o(y)$ in $G$, then the order of $\left[x, y^{\varphi}\right]$ in $\nu(G)$ divides the greatest common divisor of $o(x)$ and $o(y)$.

Using this commutator calculus and the structural information for $\nu(G)$ found in Theorem 2, we compute in [8] the nonabelian tensor square of the free nilpotent group of class 3 and rank $n$. The following group is needed to state our result.

Example 11. Let $F$ be the free group of rank $n(n-1)$ and set $\mathcal{N}$ to be $F / \gamma_{3}(F)=\left\langle y_{i, j} \mid 1 \leq i, j \leq n ; i \neq j\right\rangle$, the free nilpotent group of class two and rank $n(n-1)$. Set

$$
\begin{aligned}
& N=\left\langle\left[y_{i, j}, y_{j, i}\right],\left[y_{i, j}, y_{k, l}\right]\left[y_{i, j}, y_{l, k}\right],\left[y_{i, j}, y_{k, l}\right]\left[y_{j, i}, y_{k, l}\right],\right. \\
& {\left[y_{i, j}, y_{k, l}\right]\left[y_{l, k}, y_{j, i}\right]|1 \leq i, j, k, l \leq n ; i \neq j ; k \neq l\rangle .}
\end{aligned}
$$

Since $N \leq \mathcal{N}^{\prime} \leq Z(\mathcal{N})$, the subgroup $N$ is normal in $\mathcal{N}$. Set

$$
W_{n}=\mathcal{N} / N=\left\langle w_{i, j} \mid 1 \leq i, j \leq n ; i \neq j\right\rangle,
$$

where $w_{i, j}=y_{i, j} N$ for $1 \leq i, j \leq n$ and $i \neq j$.

Theorem 12. Let $G$ be a free nilpotent group of class 3 and rank $n$. Then $G \otimes G \cong W_{n} \times A_{f(n)}$ where $W_{n}$ is the group in Example 11 and $A_{f(n)}$ is the free abelian group of rank

$$
f(n)=\frac{n\left(3 n^{3}+14 n^{2}-3 n+10\right)}{24} .
$$

The proof of Theorem 12 does not rely on computer calculations but the identities and counting arguments found in the proof were motivated and verified by using our GAP program to compute the nonabelian tensor square for the free nilpotent groups of class 3 of rank 3,4 and 5 .

Russell Blyth and I are extending the results above to compute the nonabelian exterior square of polycyclic groups [7]. The nonabelian exterior square of a group $G$, denoted by $G \wedge G$, is the group $(G \otimes$ 
$G) / \nabla(G)$ where $\nabla(G)=\langle x \otimes x \mid x \in G\rangle$ is a central subgroup of $G \otimes G$. We denote $(g \otimes h) \nabla(G)$ by $g \wedge h$ for all $g, h$ in $G$.

Computing the nonabelian exterior square has group theoretic applications. We can compute the Schur multiplier $M(G)$ of a group $G$ from the short exact sequence

$$
0 \rightarrow M(G) \rightarrow G \wedge G \stackrel{\kappa^{\prime}}{\rightarrow} G^{\prime} \rightarrow 1,
$$

where $\kappa^{\prime}: G \wedge G \rightarrow G^{\prime}$ is defined by $\kappa^{\prime}(g \wedge h)=[g, h]$ (see [3]). The epicenter $Z^{*}(G)$ of a group $G$ is the intersection of all the central extensions of $G$. We can compute the epicenter of a group from the nonabelian exterior square in the following way. We define the exterior center $Z^{\wedge}(G)$ of a group $G$ as

$$
Z^{\wedge}(G)=\left\{a \in G \mid a \wedge g=1_{\wedge}, \forall g \in G\right\} .
$$

Theorem 13 ([13]). For any group $G$, the epicenter coincides with the exterior center, i.e. $Z^{*}(G)=Z^{\wedge}(G)$.

We say a group $G$ is capable if there exists a group $H$ such that $H / Z(H) \cong G$. The following theorem shows that computing the epicenter of a group provides a definitive test as to whether the group is capable or not.

Theorem 14 ([2]). A group $G$ is capable if and only if $Z^{*}(G)=1$.

Let $G$ and $G^{\varphi}$ be isomorphic groups via $\varphi: g \mapsto g^{\varphi}$ for all $g \in G$. Define the group $\tau(G)$ to be

$$
\begin{aligned}
\tau(G)=\left\langle G, G^{\varphi}\right|{ }^{k}\left[g, h^{\varphi}\right] & =\left[{ }^{k} g,{ }^{k} h{ }^{\varphi}\right] \\
& \left.={ }^{k^{\varphi}}\left[g, h^{\varphi}\right],\left[g, g^{\varphi}\right]=1 \forall g, h, k \in G\right\rangle .
\end{aligned}
$$

Our analysis of $\tau(G)$ follows that of $\nu(G)$. For example,

Proposition 15. Let $G$ be a group. The map $\sigma: G \wedge G \rightarrow\left[G, G^{\varphi}\right] \triangleleft$ $\tau(G)$ defined by $\sigma(g \wedge h)=\left[g, h^{\varphi}\right]$ is an isomorphism.

If $G$ is polycyclic then it follows from Theorem 6 that $G \wedge G$ and $\tau(G)$ are also. We have implemented GAP routines to compute the nonabelian exterior square of any finitely generated nilpotent group and to compute its Schur multiplier. Our computations for computing the nonabelian tensor square of the free nilpotent groups of class 3 and rank $n$ can be adjusted to compute the nonabelian exterior square of these groups. We are able to show that the epicenter of these groups are trivial. Hence they are capable. 
Our plans are to formalize our GAP programs and publish them as a GAP package. This package would compute the nonabelian tensor square and exterior square for finite groups and infinite nilpotent groups, their Schur multiplier, and the tensor and exterior centers of these groups. We also hope to generalize some of our results to a more general class of finitely generated groups than the polycyclic groups.

\section{REFERENCES}

[1] Michael R. Bacon, On the nonabelian tensor square of a nilpotent group of class two, Glasgow Math. J. 36 (1994), no. 3, 291-296. MR 95i:20054

[2] F. Rudolf Beyl, Ulrich Felgner, and Peter Schmid, On groups occurring as center factor groups, J. Algebra 61 (1979), no. 1, 161-177. MR 554857 (81i:20034)

[3] R. Brown, D. L. Johnson, and E. F. Robertson, Some computations of nonabelian tensor products of groups, J. Algebra 111 (1987), no. 1, 177202. MR 88m:20071

[4] James R. Beuerle and Luise-Charlotte Kappe, Infinite metacyclic groups and their non-abelian tensor squares, Proc. Edinburgh Math. Soc. (2) 43 (2000), no. 3, 651-662. MR 1878664 (2003d:20037)

[5] Michael R. Bacon, Luise-Charlotte Kappe, and Robert Fitzgerald Morse, On the nonabelian tensor square of a 2-Engel group, Arch. Math. (Basel) 69 (1997), no. 5, 353-364. MR 98i:20042

[6] Ronald Brown and Jean-Louis Loday, Van Kampen theorems for diagrams of spaces, Topology 26 (1987), no. 3, 311-335, With an appendix by M. Zisman. MR 88m:55008

[7] Russell D. Blyth and Robert Fitzgerald Morse, On computing the nonabelian exterior square of polycyclic groups, In preparation.

[8] _ On computing the nonabelian tensor square of polycyclic groups, To be submitted.

[9] Russell D. Blyth, Robert Fitzgerald Morse, and Joanne L. Redden, On computing the non-abelian tensor squares of the free 2-Engel groups, Proc. Edinb. Math. Soc. (2) 47 (2004), no. 2, 305-323. MR 2081056

[10] R. K. Dennis, In search of new "homology" functors having a close relationship of K-theory, Cornell University, Ithaca, N.Y. 1976.

[11] B. Eick, Ipcq - computing infinite polycyclic quotients, 2004, An Experimental GAP Package, see [16].

[12] Graham Ellis and Frank Leonard, Computing Schur multipliers and tensor products of finite groups, Proc. Roy. Irish Acad. Sect. A 95 (1995), no. 2, 137-147. MR 99h:20084

[13] Graham Ellis, On the capability of groups, Proc. Edinburgh Math. Soc. (2) 41 (1998), no. 3, 487-495. MR 1697585 (2000e:20053)

[14] B. Eick and W. Nickel, Polycyclic - computing with polycyclic groups, 2002, A GAP Package, see [16]. 
[15] Graham Ellis and Richard Steiner, Higher-dimensional crossed modules and the homotopy groups of $(n+1)-a d s$, J. Pure Appl. Algebra 46 (1987), no. 23, 117-136. MR $\mathbf{8 9 7 0 1 1}$ (88j:55010)

[16] The GAP Group, GAP - Groups, Algorithms, and Programming, Version 4.4, 2005, (http://www.gap-system.org).

[17] Aidan McDermott, Tensor products of groups and related theory, Ph.D. thesis, National University of Ireland Galway, May 1998.

[18] Clair Miller, The second homology group of a group; relations among commutators, Proc. Amer. Math. Soc. 3 (1952), 588-595. MR 0049191 (14,133c)

[19] W. Nickel, nq - nilpotent quotients of finitely presented groups, 2003, A GAP Package, see [16].

[20] N. R. Rocco, On a construction related to the nonabelian tensor square of a group, Bol. Soc. Brasil. Mat. (N.S.) 22 (1991), no. 1, 63-79. MR 1159385 (93b:20060)

[21] Nor Haniza Sarmin, Infinite two-generator groups of class two and their non-abelian tensor squares, Int. J. Math. Math. Sci. 32 (2002), no. 10, 615625. MR 1953599 (2003k:20046)

Robert Fitzgerald Morse,

Department of Electrical Engineering,

and Computer Science,

University of Evansville,

Evansville IN 47722, USA

rfmorse@evansville.edu 